\section{RMD Open}

Rheumatic \&

Musculoskeletal Diseases

\title{
Fatigue in rheumatoid arthritis; a persistent problem: a large longitudinal study
}

\author{
Hanna W van Steenbergen, ${ }^{1}$ Roula Tsonaka, ${ }^{2}$ Tom W J Huizinga, ${ }^{1}$ \\ Annelies Boonen, ${ }^{3}$ Annette $\mathrm{H}$ M van der Helm-van Mil ${ }^{1}$
}

To cite: van

Steenbergen HW, Tsonaka R, Huizinga TWJ, et al. Fatigue in rheumatoid arthritis; a persistent problem: a large longitudinal study. $R M D$ Open 2015;1:e000041. doi:10.1136/rmdopen-2014000041

- Prepublication history for this paper is available online. To view these files please visit the journal online (http://dx.doi.org/10.1136/ rmdopen-2014-000041)

Received 23 December 2014 Revised 16 February 2015 Accepted 17 February 2015

\section{$\underset{ }{\infty}$ CrossMark}

${ }^{1}$ Department of

Rheumatology, Leiden University Medical Center, Leiden, The Netherlands ${ }^{2}$ Department of Medical Statistics, Leiden University Medical Center, Leiden, The Netherlands

${ }^{3}$ Department of

Rheumatology, University Hospital Maastricht,

Maastricht, The Netherlands

Correspondence to Hanna W van Steenbergen; H.W.van_Steenbergen@lumc. $\mathrm{nl}$

\section{ABSTRACT}

Objective: Fatigue is prevalent and disabling in rheumatoid arthritis (RA). Surprisingly, the long-term course of fatigue is studied seldom and it is unclear to what extent it is influenced by inflammation. This study aimed to determine the course of fatigue during 8 years follow-up, its association with the severity of inflammation and the effect of improved treatment strategies.

Methods: 626 patients with RA included in the Leiden Early Arthritis Clinic cohort were studied during 8 years. Fatigue severity, measured on a $0-100 \mathrm{~mm}$ scale, and other clinical variables were assessed yearly. Patients included in 1993-1995, 1996-1998 and 1999-2007 were treated with delayed treatment with disease-modifying antirheumatic drugs (DMARDs), early treatment with mild DMARDs and early treatment with methotrexate respectively. After multiple imputation, the serial measurements were analysed using linear quantile mixed models.

Results: Median fatigue severity at baseline was $45 \mathrm{~mm}$ and remained, despite treatment, rather stable thereafter. Female gender (effect size $=4.4 \mathrm{~mm}$ ), younger age $(0.2 \mathrm{~mm}$ less fatigue/year), higher swollen and tender joint counts $(0.3 \mathrm{~mm}$ and $1.0 \mathrm{~mm}$ more fatigue/swollen or tender joint) and $C$ reactive protein-levels $(0.1 \mathrm{~mm}$ more fatigue per $\mathrm{mg} / \mathrm{L})$ were independently and significantly $(p<0.05)$ associated with fatigue severity over 8 years. Although improved treatment strategies associated with less severe radiographic progression, there was no effect on fatigue severity $(p=0.96)$.

Conclusions: This largest longitudinal study on fatigue so far demonstrated that the association between inflammation and fatigue is statistically significant but effect sizes are small, suggesting that non-inflammatory pathways mediate fatigue as well. Improved treatment strategies did not result in less severe fatigue. Therefore, fatigue in RA remains an 'unmet need'.

\section{INTRODUCTION}

Fatigue is common in rheumatoid arthritis (RA) and considered as one of the most important disease outcomes by patients. ${ }^{1}$ Although fatigue is not clearly defined and the cut-off for clinically relevant fatigue of

\section{Key messages}

Fatigue is a persistent problem during the course of rheumatoid arthritis.

- Persistence of inflammation is associated with persistence of more severe fatigue.

- Improved treatment strategies have not resulted in less severe fatigue during the course of rheumatoid arthritis.

most fatigue measures is unknown, the prevalence of fatigue in RA has been reported to be $40-80 \% .^{23}$ The majority of studies evaluating fatigue in RA had cross-sectional designs or studied patients with RA with different disease durations; therefore little is known on the long-term course of fatigue. ${ }^{4}$

The importance of fatigue in RA is underlined by the finding that more severe fatigue is predictive for decreased physical and mental health-related quality of life, depression and loss of work ability. ${ }^{5-7}$ Both a Patient Perspective Workshop at OMERACT (Outcome Measures in Rheumatology) in 2007 and a EULAR/ACR task force in 2008 recommended that 'each trial should report on fatigue'. 9 Furthermore, since severe radiographic joint destruction (the traditional outcome measure in trials) is no longer prevalent, other outcomes will become more important. In the light of these developments, it is surprising that so little is known about the fatigue severity during the disease course.

The causation of fatigue in RA is thought to be multidimensional. A recently proposed conceptual model suggests that fatigue is the result of interactions between three factors: disease-process related factors, cognitive and behavioural factors (thoughts, feelings, behaviours) and personal factors (personal life issues) ${ }^{10}$ Scientific data supporting this model are mostly derived from cross-sectional studies and are conflicting. ${ }^{4} \mathrm{~A}$ recent systematic literature review concluded that none of the 
studied associations were consistent across all published data. ${ }^{4}$ Most consistent were the associations of fatigue severity with the degree of pain and physical impairments. To a lesser extent, there was some consistency in the results showing that depressive mood/depression and female sex associated with more severe fatigue. $^{2} 4{ }^{11-15}$ In contrast, the relation between markers of inflammation and fatigue remained disputable as contradictory results were obtained on the associations between fatigue and erythrocyte sedimentation rate, $\mathrm{C}$ reactive protein (CRP), joint counts and disease activity scores. ${ }^{2}{ }^{111-15}$ Thus, although intuitively it is generally felt that fatigue in RA is in part the consequence of inflammation (either clinically apparent or subclinical inflammation), the question whether and to what extent fatigue in RA is mediated by inflammation remains unanswered.

This study aimed to assess (1) whether fatigue severity differed at disease onset between patients presenting with RA and other forms of early arthritis. Within RA, a large longitudinal 8 years study on fatigue was performed aiming to determine (2) the course of fatigue, (3) the association between the course of inflammation and the course of fatigue and (4) the effect of improved treatment strategies, which have resulted in better suppression of disease activity, on the course of fatigue.

\section{PATIENTS AND METHODS Longitudinal cohort}

All patients were included in the Leiden Early Arthritis Clinic cohort, a population-based inception cohort in the Netherlands that started in 1993 and has been described in detail previously. ${ }^{16}$ In short, inclusion took place when arthritis was confirmed at physical examination and symptom duration was $<2$ years. At baseline, rheumatologists completed questionnaires, 66-swollen and 68-tender joint counts (SJC and TJC) were performed, patients filled out questionnaires among which the Health Assessment Questionnaire (HAQ), hand and feet radiographs were taken, as well as blood samples for routine laboratory screening (including CRP, haemoglobin ( $\mathrm{Hb})$, immunoglobulin ( $\mathrm{Ig})$ M-rheumatoid factor (RF) and anticitrullinated peptide antibodies (ACPA) (anti-CCP2, Eurodiagnostica, the Netherlands). Written informed consent was obtained from all patients. The study was approved by the local Medical Ethics Committee.

To measure fatigue severity, patients were asked by the research nurses to note the point that best reflected the fatigue they had experienced during the last day. ${ }^{17}$ This was expressed on a visual analog scale (VAS) ranging from 0 (no fatigue) to $100 \mathrm{~mm}$ (extreme fatigue) for the patients assessed between 1993 and 2010 and on a numerical rating scale (NRS) ranging from 0 (no fatigue) to 10 (extreme fatigue) from 2010 onwards, when digital recording forms were introduced. Since the
VAS and NRS are known to correlate strongly, ${ }^{18} 19$ results of the NRS were multiplied by 10 .

Follow-up visits were performed yearly and included standard clinical assessment including joint counts, rating the fatigue severity, $\mathrm{HAQ}$ and radiographs of hands and feet. Serial radiographs were scored with known time-order by one experienced reader using Sharp-van-der-Heijde scores blinded to any clinical data (intraclass-correlation coefficient 0.91), as described previously. ${ }^{16}$ The initial treatment strategy of patients with RA has changed over time. Generally, patients included in 1993-1995 were initially treated with nonsteroidal anti-inflammatory drugs (NSAIDs) and diseasemodifying antirheumatic drugs (DMARDs) were initiated with delay, patients included in 1996-1998 were early treated with mild DMARDs (mainly hydroxychloroquine or sulphasalazine) and patients included in 1999-2007 were early treated with methotrexate. In addition, since 1999 DMARD-therapy was more rapidly adjusted in case of insufficiently suppressed disease activity score (DAS-steered treatment).

\section{Patient selection}

Between 1993 and March 2013 in total 3112 early patients with arthritis with different diagnoses were included in the EAC. Of these patients, 2442 (78.5\%) had baseline fatigue data. Patients with and without baseline fatigue data did not differ in age, gender, SJC, TJC, or frequency of ACPA-positivity or having RA; patients with missing baseline fatigue data had slightly higher CRP-levels (median (IQR) $12 \mathrm{mg} / \mathrm{L}$ (4-37) versus $10 \mathrm{mg} / \mathrm{L}$ (3-27), $\mathrm{p}=0.002)$. All 2442 early patients with arthritis were studied to compare fatigue at disease onset across different diagnoses.

Of the 2442 early patients with arthritis included between 1993 and 2013, 902 patients fulfilled the 1987-ACR criteria for RA. Associations of baseline characteristics with fatigue at disease onset in RA were studied in these 902 patients.

Longitudinal studies on yearly measured fatigue were done on the 626 patients with RA included before 2007, providing the possibility to have completed 8 years follow-up. Similar as for fatigue, SJC, TJC, CRP, Hb, HAQ and SHS were measured yearly, providing the opportunity to test associations between fatigue and the concomitantly measured clinical variables during follow-up. In other words, this allowed studying the course of other variables in relation to the course of fatigue. Furthermore, in these patients the effects of improved treatment strategies in RA on three different long-term outcomes were studied, namely radiographic progression, SJC and fatigue over time.

A large majority of patients without RA (eg, patients with reactive arthritis, inflammatory osteoarthritis or paramalignant arthritis) were not followed for many years. Patients diagnosed with undifferentiated arthritis (UA), psoriatic arthritis/spondylarthropathy with 
peripheral arthritis (PsA/SpA), (pseudo)gout and systemic connective tissue disease (SCTD) often did not complete 8 years follow-up but considerable proportions completed 3 years follow-up. In these patients, available fatigue data were evaluated to determine whether the 3 years course of fatigue was different in RA compared to other forms of chronic arthritis.

\section{Statistical analyses}

As fatigue severity was non-normally distributed, median levels were presented and modelled. Cross-sectional quantile regression analyses were used for the comparisons on baseline fatigue data (comparisons across different diagnoses and within RA). For the longitudinal 8-year analyses of fatigue within RA linear quantile mixed models were used ${ }^{20}{ }^{21}$ modelling the course of medians over time instead of means as in linear models. ${ }^{22}$ SJC, TJC, CRP, Hb, SHS and HAQ were measured yearly and not constant over time and analysed for its association with fatigue over time as time-dependent variables. Age, gender, ACPA and RF reported or measured at baseline were analysed. Before performing the analyses within RA, multiple imputation was used to deal with missing data for fatigue severity, symptom duration, duration of morning stiffness, SJC, TJC, CRP, Hb, RF, ACPA, SHS and HAQ. Five data sets were created for both the analyses of associations with fatigue at disease onset within RA and for the repeated measurements over 8 years by using multivariate imputation by chained equations that generates multiple imputations for incomplete multivariate data by Gibbs sampling. ${ }^{23}$

The association between the different treatment strategies (reflected by the proxy different inclusion period) and radiographic progression was analysed over 8 years using a multivariate normal regression analysis adjusted for age and gender, as described in detail previously. ${ }^{24} 25$ A linear quantile mixed regression analysis was used to analyse whether different treatment strategies associated with the number of swollen joints over 8 years.

Analyses were performed using $\mathrm{R}$ statistical software package $^{2627}$ and SPSS V.20.0. p Values $<0.05$ were considered significant.

\section{RESULTS}

\section{Baseline fatigue severity across different forms} of early arthritis

Table 1 presents the characteristics of the studied patients. First, we studied whether the fatigue severity differed between patients presenting with RA and other forms of early arthritis. Of the 2442 patients with fatigue data at disease onset, 902 patients had RA and 1540 patients other diagnoses. Figure 1A presents medians of fatigue severity for different diagnoses. Patients with SCTD and RS3PE recorded significantly more severe fatigue than patients with RA (medians respectively

Table 1 Baseline characteristics

\begin{tabular}{|c|c|c|c|}
\hline & $\begin{array}{l}\text { Patients with RA with } \\
\text { fatigue data at disease } \\
\text { onset }(n=902)^{\star}\end{array}$ & $\begin{array}{l}\text { Non-RA early patients with } \\
\text { arthritis with fatigue data } \\
\text { at disease onset }(n=1540) \dagger\end{array}$ & $\begin{array}{l}\text { Patients with } R A \text { with } \\
\text { repeated fatigue data over } \\
8 \text { years }(n=626) \ddagger\end{array}$ \\
\hline $\begin{array}{l}\text { Age at disease onset, } \\
\text { mean (SD), years }\end{array}$ & $56.6(15.3)$ & $49.9(17.4)$ & $56.5(15.5)$ \\
\hline Female, n (\%) & 607 (67.3) & $868(56.4)$ & $429(68.5)$ \\
\hline Inclusion period & 1993-2013 & 1993-2013 & 1993-2007 \\
\hline $\begin{array}{l}\text { Symptom duration, } \\
\text { median (IQR), weeks }\end{array}$ & $18.3(9.3-34.7)$ & $13.1(5.1-29.1)$ & $19.3(10.7-39.3)$ \\
\hline $\begin{array}{l}\text { Morning stiffness, } \\
\text { median (IQR), minutes }\end{array}$ & $60(30-120)$ & $30(0-60)$ & $60(30-120)$ \\
\hline SJC, median (IQR) & $7(4-12)$ & $2(1-6)$ & $8(4-14)$ \\
\hline TJC, median (IQR) & $4(7-10)$ & $4(2-7)$ & $8(5-12)$ \\
\hline CRP, median (IQR), mg/L & $14(6-33)$ & $8(3-23)$ & $17(8-39)$ \\
\hline $\mathrm{Hb}$, mean (SD), mmol/L & $8.2(0.8)$ & $8.4(0.9)$ & $8.1(0.8)$ \\
\hline ACPA-positive, n (\%) & $456(52.1)$ & $105(9.1)$ & $326(54.1)$ \\
\hline RF-positive, n (\%) & $516(57.9)$ & $228(15.1)$ & $364(59.2)$ \\
\hline SHS, median (IQR) & $5(2-11)$ & N/A & $5(2-11)$ \\
\hline HAQ, median (IQR) & $1(0.6-1.5)$ & $0.6(0.3-1.0)$ & $1(0.6-1.5)$ \\
\hline \multicolumn{4}{|c|}{$\begin{array}{l}\text { *Symptom duration was missing in } 54 \text { patients, morning stiffness in } 78 \text { patients, SJC in } 14 \text { patients, TJC in } 236 \text { patients, CRP in } 30 \text { patients, } \\
\text { Hb in } 18 \text { patients, ACPA-status in } 27 \text { patients, RF-status in } 11 \text { patients, SHS in } 372 \text { patients and HAQ in } 108 \text { patients. } \\
\text { †Symptom duration was missing in } 113 \text { patients, morning stiffness in } 188 \text { patients, SJC is } 37 \text { patients, TJC in } 467 \text { patients, CRP in } \\
78 \text { patients, Hb in } 43 \text { patients, ACPA-status in } 392 \text { patients, RF-status in } 31 \text { patients and HAQ in } 221 \text { patients. The radiographs of the patients } \\
\text { without RA were not SHS scored. } \\
\text { fSymptom duration was missing in } 37 \text { patients, morning stiffness in } 19 \text { patients, SJC in } 4 \text { patients, TJC in } 334 \text { patients, CRP in } 36 \text { patients, } \\
\text { The missing values were imputed for the analyses of associations between fatigue and other variables within RA. } \\
\text { Hb in } 16 \text { patients, ACPA-status in } 23 \text { patients, RF-status in } 11 \text { patients, SHS in } 19 \text { patients and HAQ in } 104 \text { patients. } \\
\text { ACPA, anticitrullinated peptide antibody; CRP, C reactive protein; HAQ, Health Assessment Questionnaire; Hb, haemoglobin; } \\
\text { N/A, not applicable; RA, rheumatoid arthritis; RF, IgM rheumatoid factor; SHS, Sharp-van der Heijde score; SJC, } 66 \text {-swollen joint count; } \\
\text { TJC, 68-tender joint count. }\end{array}$} \\
\hline
\end{tabular}




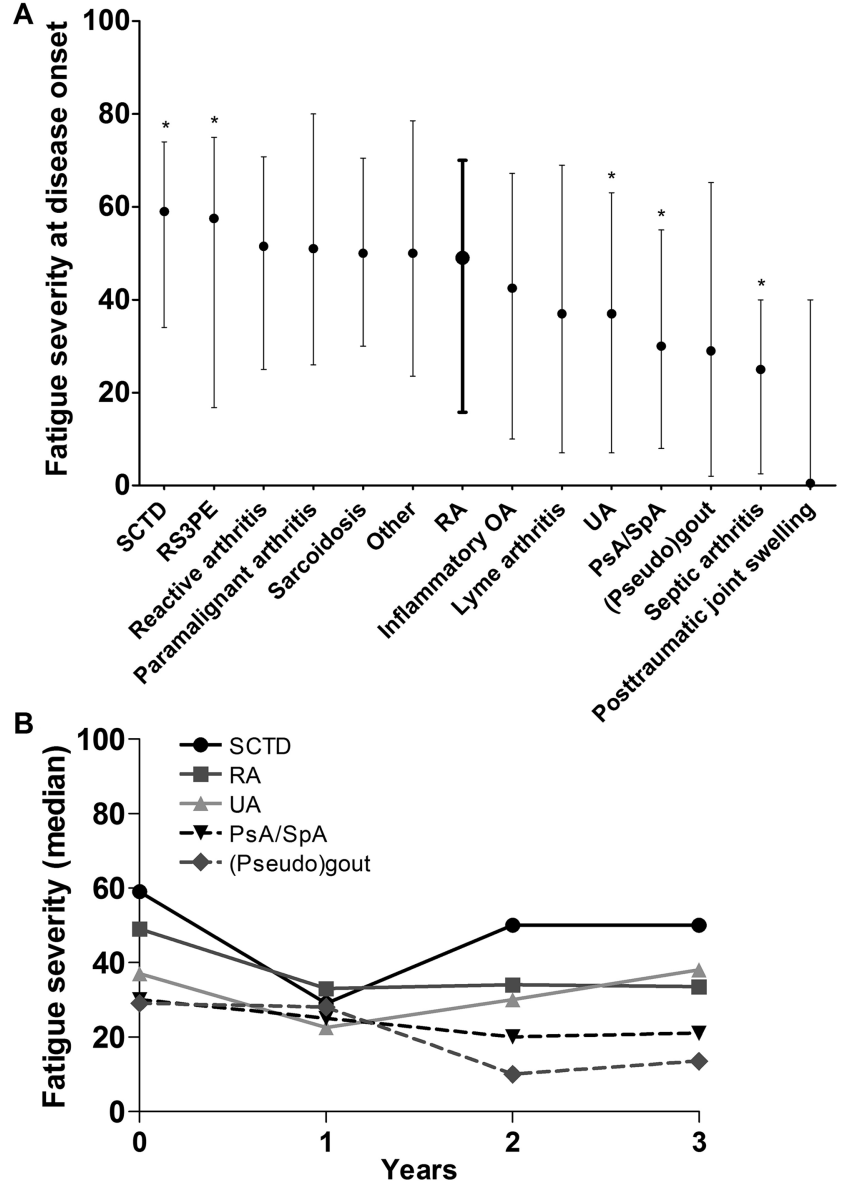

Figure 1 Fatigue severity across early patients with arthritis with different diagnoses at disease onset $(A)$ and over 3 years of disease (B). (A) Presented are medians and IQRs of fatigue severity at disease onset. The data of rheumatoid arthritis (RA) are presented in bold. An asterisk indicates a significant different fatigue level compared to RA when adjusted for age and gender. The numbers of patients at baseline are 902 for RA, 73 for SCTD, 48 for RS3PE, 96 for reactive arthritis, 19 for paramalignant arthritis, 65 for sarcoidosis, 25 for others, 126 for inflammatory OA, 13 for lyme arthritis, 706 for UA, 271 for PsA/SpA, 90 for (pseudo) gout, four for septic arthritis and four for post-traumatic joint swelling. (B) Presented are medians of fatigue severity over 3 years of disease. Available, unmodelled data without imputation of missing data is depicted. The numbers of available fatigue data per diagnosis at baseline, one, 2 and 3 years follow-up were respectively: $73,32,25$ and 21 for SCTD; 902, 537, 411 and 432 for RA; 706, 270, 155 and 139 for UA; 271, 151, 110, 101 for PsA/SpA; 90, 13, 4 and 2 for (pseudo)gout. SCTD, systemic connective tissue disease; RS3PE, remitting seronegative symmetrical synovitis with pitting edema; RA, rheumatoid arthritis; OA, osteoarthritis; UA, undifferentiated arthritis; PsA, psoriatic arthritis;

$\mathrm{SpA}$, spondylarthropathy with peripheral arthritis.

$59 \mathrm{~mm}$ and $58 \mathrm{~mm}$ compared to $49 \mathrm{~mm}$ in RA; $\mathrm{p}<0.05$ adjusted for age and gender). Patient groups that experienced significantly less fatigue than patients with RA were patients with UA (median $37 \mathrm{~mm}$ ), PsA/SpA (median $30 \mathrm{~mm}$ ) and septic arthritis $(25 \mathrm{~mm})$ (all $\mathrm{p}<0.05$ adjusted for age and gender). Four included patients were finally diagnosed with post-traumatic joint swelling; these patients had a median fatigue severity of zero (figure 1). Evaluating available data on the fatigue course over the first 3 years of disease revealed a similar trend as for the baseline data: patients with SCTD remained more severe fatigued than patients with RA and patients with PsA/SpA and (pseudo)gout had less severe fatigue also during follow-up (figure 1B).

\section{Baseline fatigue severity in relation to baseline characteristics in RA}

Within the 902 patients with RA it was studied whether fatigue at disease onset associated with other baseline characteristics (table 2). Independent associations with more severe fatigue were observed for female $(p=0.001)$,

Table 2 Fatigue severity at disease onset in relation to clinical variables at disease onset in rheumatoid arthritis

\begin{tabular}{|c|c|c|}
\hline & $\begin{array}{l}\text { Effect size } \\
\text { in } \mathrm{mm} \text { (SE) }\end{array}$ & p Value \\
\hline \multicolumn{3}{|l|}{ Univariable analyses } \\
\hline Female & $7.1(3.4)$ & $0.040^{*}$ \\
\hline Age at onset, per year & $-0.004(0.1)$ & 0.95 \\
\hline Symptom duration, per week & $0.1(0.02)$ & $<0.001^{*}$ \\
\hline $\begin{array}{l}\text { Morning stiffness duration, } \\
\text { per minute }\end{array}$ & $0.04(0.01)$ & $<0.001^{*}$ \\
\hline SJC, per joint & $0.4(0.2)$ & $0.010^{\star}$ \\
\hline TJC, per joint & $1.4(0.2)$ & $<0.001^{*}$ \\
\hline CRP, per mg/L & $0.1(0.05)$ & 0.072 \\
\hline $\mathrm{Hb}$, per $\mathrm{mmol} / \mathrm{L}$ & $-3.6(1.2)$ & $0.002^{*}$ \\
\hline ACPA-positivity & $-4.3(3.0)$ & 0.15 \\
\hline RF-positivity & $-0.3(2.4)$ & 0.90 \\
\hline SHS, per point & $0.02(0.2)$ & 0.93 \\
\hline \multicolumn{3}{|l|}{ Multivariable analysis } \\
\hline Female & $7.6(2.3)$ & $0.001^{*}$ \\
\hline Age at onset, per year & $-0.01(0.1)$ & 0.20 \\
\hline Symptom duration, per week & $0.1(0.02)$ & $<0.001^{*}$ \\
\hline $\begin{array}{l}\text { Morning stiffness duration, } \\
\text { per minute }\end{array}$ & $0.03(0.01)$ & $0.002^{*}$ \\
\hline SJC, per joint & $-0.3(0.2)$ & 0.071 \\
\hline TJC, per joint & $1.1(0.2)$ & $<0.001^{*}$ \\
\hline CRP, per mg/L & $0.1(0.03)$ & 0.096 \\
\hline $\mathrm{Hb}$, per $\mathrm{mmol} / \mathrm{L}$ & $-0.9(1.5)$ & 0.54 \\
\hline ACPA-positivity & $-7.9(2.6)$ & $0.003^{*}$ \\
\hline RF-positivity & $3.8(2.7)$ & 0.15 \\
\hline
\end{tabular}

${ }^{*} \mathrm{p}<0.05$

Presented are the results of the analyses on 902 patients with RA with fatigue severity at disease onset as outcome and the other variable at disease onset as independent variable. Variables with $p$ values $<0.05$ in univariable analyses and clinically relevant variables were included in multivariable analysis. The effect sizes indicate how much the fatigue severity at disease onset on a 0-100 $\mathrm{mm}$ scale changed with 1 unit increase in the other variable at disease onset. For example, women have a $7.1 \mathrm{~mm}$ higher fatigue severity at baseline compared to men and the fatigue severity measured at disease onset increased $1.4 \mathrm{~mm}$ per tender joint.

ACPA, anticitrullinated peptide antibodies; CRP, C reactive protein; $\mathrm{Hb}$, haemoglobin; RA, rheumatoid arthritis; RF, IgM rheumatoid factor; SHS, Sharp-van der Heijde score; SJC, 66-swollen joint count; TJC, 68-tender joint count. 
patients with more morning stiffness $(p=0.002)$, more tender joints $(\mathrm{p}<0.001)$ and patients without ACPA $(\mathrm{p}=0.003)$. Also longer symptom duration at disease presentation associated with more severe fatigue at the first visit $(p<0.001)$. Additionally, patients that reported a higher level of disability (HAQ) reported also more severe fatigue $(20.5 \mathrm{~mm}$ increase in fatigue severity with 1 point increase in $\mathrm{HAQ}, \mathrm{p}<0.001)$.

\section{Course of fatigue in RA}

626 patients with RA were studied during 8 years follow-up. At baseline, the median (IQR) fatigue severity was $45 \mathrm{~mm}(18-65 \mathrm{~mm})$ and, despite initiation of treatment, the fatigue severity slightly decreased during the first year but then remained rather stable (figure 2).

\section{Course of fatigue in relation to course of inflammation in RA—univariable analyses}

Measures of inflammation (SJC, TJC and CRP) changed during the disease course as result of either the disease itself or the applied intervention. Similar to fatigue, these markers were measured yearly, allowing determining associations between the course of inflammation and the course of fatigue during 8 years. Patients that over time remained to have higher number of swollen joints, tender joints and CRP-levels also remained more fatigued (all $\mathrm{p}<0.001$ ). The observed effect sizes of the inflammatory markers were all small. For example, presence of one additional swollen joint over time associated with $0.7 \mathrm{~mm}$ more fatigue over time and patients with $1 \mathrm{mg} / \mathrm{L}$ higher CRP over time reported $0.1 \mathrm{~mm}$ more fatigue (table 3).

Although we aimed to evaluate whether the course of inflammation associated with the course of fatigue, some other clinical variables that might be of importance

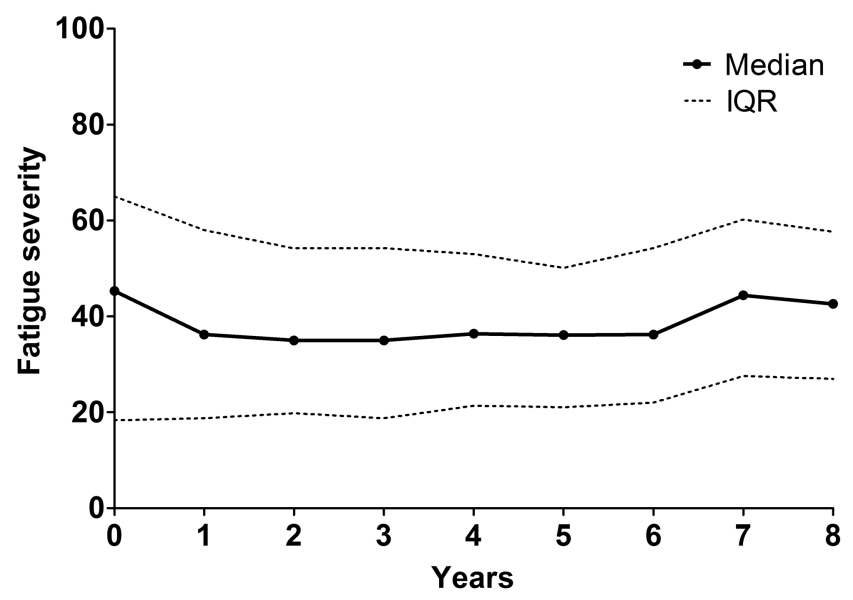

Figure 2 The severity of fatigue over 8 years of disease in early rheumatoid arthritis patients. Presented are the median values with IQR of fatigue severity in 626 early patients with RA with missing data imputed. The numbers of patients with available data per year were: 510 for baseline, 350 for year 1 , 298 for year 2, 280 for year 3, 266 for year 4, 251 for year 5 , 208 for year 6,192 for year 7 and 166 for year 8 .
Table 3 Fatigue severity over 8 years in relation to the course of inflammation and other variables in rheumatoid arthritis

\begin{tabular}{lcc}
\hline & $\begin{array}{c}\text { Effect size } \\
\text { in } \mathbf{~ m m ~ ( S E ) ~}\end{array}$ & p Value \\
\hline Univariable analyses & & \\
Female & $6.1(1.7)$ & $<0.001^{*}$ \\
Age at disease onset, per year & $-0.2(0.1)$ & $0.043^{*}$ \\
SJC, per joint & $0.7(0.1)$ & $<0.001^{*}$ \\
TJC, per joint & $1.1(0.1)$ & $<0.001^{*}$ \\
CRP, per mg/L & $0.1(0.03)$ & $<0.001^{*}$ \\
Hb, per mmol/L & $-3.6(1.2)$ & $0.003^{*}$ \\
ACPA-positivity & $-0.5(2.0)$ & 0.79 \\
RF-positivity & $-3.3(1.7)$ & 0.053 \\
SHS, per point & $-0.1(0.1)$ & 0.14 \\
Multivariable analysis & & \\
Female & $4.4(1.9)$ & $0.022^{*}$ \\
Age at disease onset, per year & $-0.2(0.1)$ & $0.016^{*}$ \\
SJC, per joint & $0.3(0.1)$ & $0.022^{*}$ \\
TJC, per joint & $1.0(0.1)$ & $<0.001^{*}$ \\
CRP, per mg/L & $0.1(0.04)$ & $0.049^{*}$ \\
Hb, per mmol/L & $-0.9(1.1)$ & 0.38 \\
ACPA-positivity & $0.4(2.1)$ & 0.84 \\
RF-positivity & $-1.9(2.1)$ & 0.36 \\
\hline Presented are the restls
\end{tabular}

Presented are the results of the longitudinal analyses in 626 patients with RA. Variables with $p$ values $<0.05$ in univariable analysis and clinically relevant variables were included in multivariable analysis. The outcome (fatigue) and the clinical variables SJC, TJC, CRP, $\mathrm{Hb}$ and SHS were measured yearly. Gender, age, RF and ACPA were determined at disease onset. The effect sizes indicate how much the fatigue on a $0-100 \mathrm{~mm}$ scale change with 1 unit increase in the other variable. For example, $1 \mathrm{mg} / \mathrm{L}$ increase in CRP is associated with a $0.1 \mathrm{~mm}$ increase in fatigue severity measured at the same time point during 8 years follow-up and women have a $6.1 \mathrm{~mm}$ higher fatigue score at every time point over 8 years than men.

ACPA, anticitrullinated peptide antibodies; CRP, C reactive protein; $\mathrm{Hb}$, haemoglobin; RA, rheumatoid arthritis; RF, IgM rheumatoid factor; SHS, Sharp-van der Heijde score;

SJC, 66-swollen joint count; TJC, 68-tender joint count.

when evaluating fatigue severity were studied. Female and younger patients remained more fatigued over time (females $6.1 \mathrm{~mm}$ more severe fatigue than males, $\mathrm{p}<0.001 ; 0.2 \mathrm{~mm}$ less severe fatigue per year older, $\mathrm{p}=0.043$, table 3 ). The course of fatigue was similar for patients with and without RA-related autoantibodies. Patients with lower Hb-levels during the disease course remained more fatigued $(p=0.003)$.

Furthermore, patients with more functional impairment during the disease course (higher HAQ-scores), were also more severe fatigued; per point increase in HAQ the fatigue severity increased with $14.6 \mathrm{~mm}$ $(p<0.001)$. (Figures that present fatigue scores over time in relation to some characteristics are available from the corresponding author on request).

\section{Course of fatigue in relation to course of inflammation in RA-multivariable analysis}

Characteristics with $\mathrm{p}<0.05$ in univariable analyses and clinically relevant variables were included in multivariable analysis. This revealed that higher SJC $(p=0.022$, 
$0.3 \mathrm{~mm}$ more fatigue per swollen joint), higher TJC $(\mathrm{p}<0.001,1.0 \mathrm{~mm}$ more fatigue per tender joint $)$ and higher CRP $(\mathrm{p}=0.049,0.1 \mathrm{~mm}$ more fatigue per $\mathrm{mg} / \mathrm{L}$ CRP) (table 3) associated with more severe fatigue over time, indicating a significantly independent association of the severity of inflammation over time with persistence of more severe fatigue. Also female ( $p=0.022$, difference with males $4.4 \mathrm{~mm}$ ) and younger patients ( $\mathrm{p}=0.016,0.2 \mathrm{~mm}$ lower scores per year older) reported more severe fatigue.

\section{Improved treatment strategies and the course of fatigue in RA}

In general, improved treatment strategies in RA have resulted in improved disease outcomes. To validate this notion in present data set, we explored the association of the described treatment strategies with the severity of radiographic progression. A significant difference in radiographic progression was observed between the three groups: with delayed DMARD-treatment as reference (inclusion 1993-1995), patients early treated with mild DMARDs (inclusion 1996-1998) had 0.97-fold less severe radiographic progression per year $(\mathrm{p}=0.026)$ and patients early treated with methotrexate followed by DAS-steered treatment (inclusion 1999-2007) had 0.92-fold less severe radiographic progression per year $(\mathrm{p}<0.001$, figure $3 \mathrm{~A})$. We also evaluated whether the median number of swollen joints over time was different for the patients with RA treated with different treatment strategies. Indeed, improved treatment strategies associated with a reduction in SJC during disease course: with the delayed treatment group (1993-1995) as reference, patients early treated with mild DMARDs (1996-1998) had 1.4 less swollen joints $(\mathrm{p}=0.005)$ over 8 years and patients early treated with methotrexate (1999-2007) had 3.6 less swollen joints over 8 years $(\mathrm{p}<0.001)$ (figure $3 \mathrm{~B})$. In line with these observations, we hypothesised that improved treatment strategies also associated with a less severe fatigue course. However, no univariable association was observed. Patients with RA early treated with mild DMARDs or methotrexate did not experience less severe fatigue over time compared to patients treated with initial treatment with NSAIDs and delayed DMARD-therapy $(p=0.80$ and $\mathrm{p}=0.79$, respectively, figure $3 \mathrm{C}$ ). This indicates that despite improved treatment strategies and subsequent decreased inflammation-levels during the disease course, the fatigue severity in RA remained unchanged.

\section{DISCUSSION}

Although fatigue in RA is convincingly known to be associated with functional loss and is considered an important outcome measure for clinical trials, ${ }^{8} 9$ little is known on its long-term course. Furthermore, the results of existing studies on the causation of fatigue were conflicting with regards to whether fatigue is associated with inflammatory markers. This large longitudinal study evaluated the course of fatigue in RA and observed that
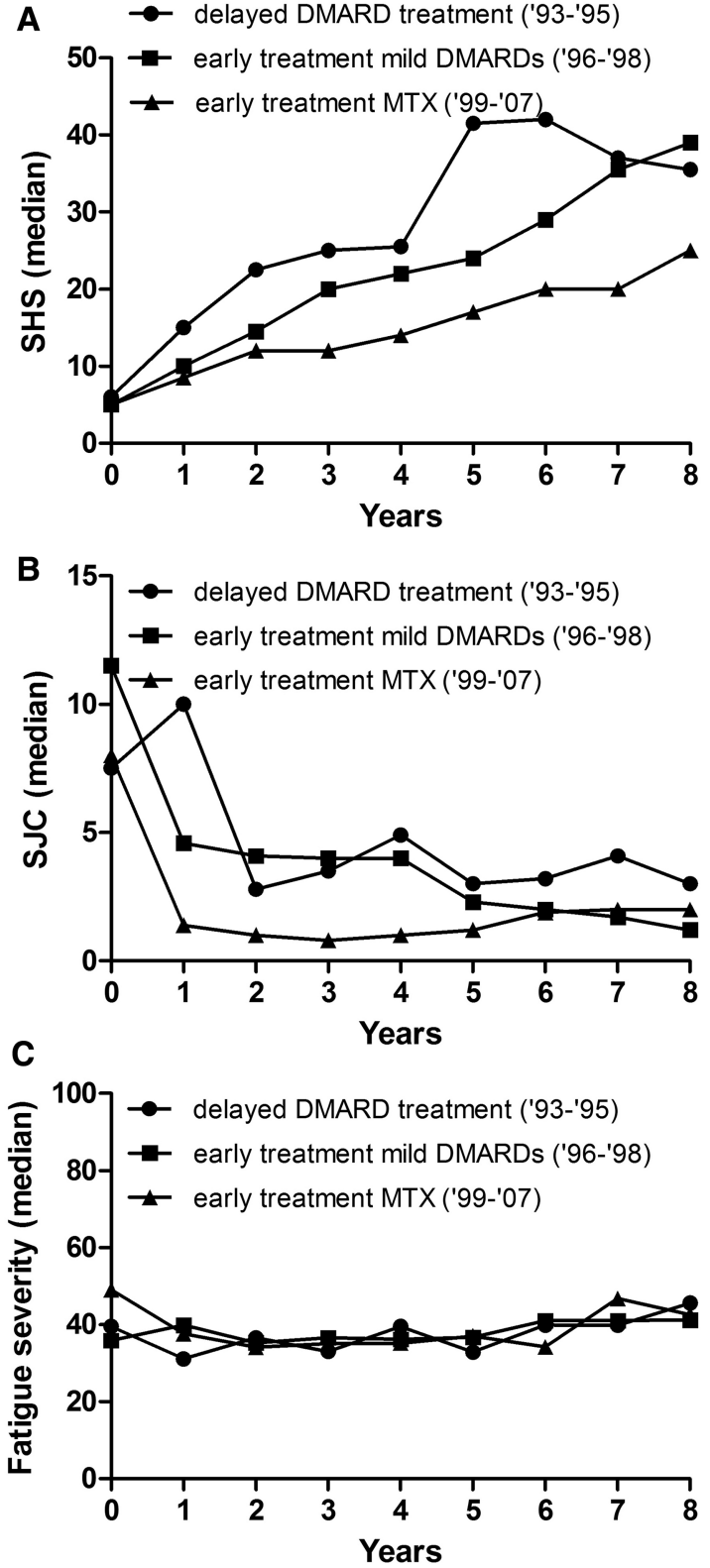

Figure 3 Different treatment strategies in rheumatoid arthritis in relation to radiographic progression $(A)$ number of swollen joints $(B)$ and fatigue severity over time $(C)$.

Presented are three long-term outcomes in relation to treatment strategies. Treatment strategies are reflected by different inclusion periods as the initial treatment strategy differed for different inclusion periods. The inclusion period 1993-1995 comprised 100 patients, 1996-1998 166 patients and 1999-2007 360 patients. Radiographic progression: 1993-1995=reference, 1996-1998 $\beta=0.97 p=0.026$; 1999$2007 \beta=0.92 p<0.001$. The $\beta$ indicates the fold rate of joint destruction per year compared to the reference. Swollen joint count: 1993-1995=reference, 1996-1998 effect size $=-1.4$ $p=0.005 ; 1999-2007$ effect size $=-3.6 p<0.001$, omnibus test for overall significance of model $p<0.001$. The effect size indicates the difference in number of swollen joints compared to the reference. Fatigue severity: 1993-1995=reference, 1996-1998 $p=0.80 ; 1999-2007 p=0.79$; omnibus test for overall significance of model $p=0.96$. SHS, Sharp-van der Heijde score; SJC, swollen joint count; DMARD, disease-modifying antirheumatic drugs. 
patients with higher inflammatory markers (more tender/swollen joints, higher CRP-level) over time also experienced more severe fatigue over time. Hence, persistence of inflammation is statistically significant associated with more severe fatigue, but the observed effect sizes were small. Furthermore, improved treatment strategies did not result in a less severe fatigue course.

Previous studies evaluating fatigue in RA observed inconsistent results on whether inflammation associated with fatigue. The majority of these studies had crosssectional study designs or short follow-up ${ }^{4}$ and were presumably underpowered to observe significance for small effects. Present study was powerful because of the repeated measurements during 8 years follow-up and by the use of a statistical method that takes advantage of the correlation between the serial measurements. Although we clarified that a relation between persistent inflammation and persistent fatigue is present, the question is whether the observed effect sizes are clinically relevant. The minimal clinically important difference (MCID) for the VAS fatigue, which is the smallest improvement in score that is perceived as beneficial by patients, has been reported to be around $10 \mathrm{~mm}^{28}$ and the unadjusted effect size per swollen joint was $0.7 \mathrm{~mm}$, indicating that per five additional swollen joints, fatigue severity increased with $3.5 \mathrm{~mm}$ on a $0-100 \mathrm{~mm}$ scale. Although the observed effect sizes were small, when a combination of characteristics is present, for instance several tender/ swollen joints and increased CRP, the limit of a clinically meaningful difference of fatigue is reached.

Based on the observed association between inflammation and fatigue and on the efficacy of improved treatment strategies on both inflammation and radiographic progression, we hypothesised that these treatment strategies had resulted also in less severe fatigue. The inclusion periods were used as proxies for treatment strategy. A limitation is that other (unmeasured) factors changed over time as well. Nonetheless, patients with RA treated according to nowadays regimens experienced equally severe fatigue as patients initially treated with NSAIDs and initiation of DMARDs with delay. This finding might be unexpected as the latter patient group had less severe inflammation over time (for instance lower SJCs) and we had observed that less inflammation associated with less severe fatigue. Apparently other factors with contrary effects on fatigue were present as well.

We then hypothesised that patients treated with nowadays regimens and consequently with less inflammation during the disease, rated their fatigue higher and therefore the decrease in levels of inflammation was not accompanied by a decrease in fatigue severity. To gain support for this hypothesis, an additional multivariable analysis including inflammatory markers and the applied treatment strategy (inclusion period) was performed. This revealed that when adjusted for inflammatory measures, patients treated in most recent treatment group (inclusion 1999-2007) had indicated significantly more severe fatigue compared to patients included in

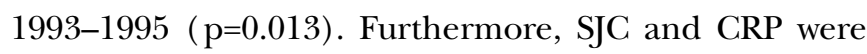
not significantly associated anymore (data are available from the corresponding author on request). This result is in line with that of Putrik et $a l^{29}$ who observed that patients with RA in countries with higher welfare scored worse on patients-reported outcomes like fatigue despite lower levels of inflammation. Also in line is a study that compared patients with RA in 1985 and 2000, showing that improvement in objectively measured disease outcomes was not accompanied by improvement in patientreported pain. ${ }^{30}$ Although causality can never be proved using association analyses, a similar effect might explain the persistence of fatigue despite decreased levels of inflammation. Present results, at least illustrate the multidimensional origin of fatigue and imply that noninflammatory pathways are relevant as well. Consequently, other types of intervention should be initiated to reduce the disease burden caused by fatigue. ${ }^{31}$

Notably, biologics were rarely used in the patients with RA studied here $(<5 \%)^{24}$; other studies are needed to determine whether treatment with biologics differently affect fatigue severity in RA.

We observed more severe fatigue in female patients with RA. This is in line with results obtained in other studies. ${ }^{4}$ Sex differences in RA have been reported not only for fatigue but also for other patient-reported outcomes like pain, function and global status with female having worse scores. ${ }^{32} 33$

The fatigue data were non-normally distributed. To prevent that a small proportion of patients disproportionally affected the study results, quantile regression analyses were performed. This method models medians instead of means and is more robust to outliers. Over time patients had missing data, both on fatigue and other variables. Since these missing values were not completely at random, analyses of available data may introduce bias $^{34}$ and therefore, multiple imputation was performed. However, no large differences are seen in the 8-year course of fatigue when using available data only and after imputation of missing values (data are available from the corresponding author on request).

We performed analyses on group level. We realise that this inflicts simplicity of reality because changes of individuals are averaged. However, presumably, there are individual patients with a remarkable decrease in fatigue severity during disease course (in response to treatment or otherwise), while others may have stable or increasing levels of fatigue. To get some insight in this assumption, we evaluated fatigue severity on patient level during the first 2 years of disease. It was observed that during the first and second year of disease, respectively $49 \%$ and $62 \%$ of the patients had rather stable fatigue levels while the others had an increase or decrease in fatigue severity. Furthermore, it was observed that the patients with most severe fatigue were partly different at different time-points (data are available from the corresponding author on request). This indicates that on patient level fatigue severity can differ during the disease course 
and that on group level fatigue remains a persistent problem in RA.

We measured fatigue severity using a VAS. Several other fatigue measures are available; these are multi-item or multidimensional measures that explore broader fatigue issues or various domains of fatigue. Such multidimensional measures are useful for exploring fatigue causality and evaluation of fatigue interventions. Compared to such tools, a VAS provides a one-dimensional assessment of fatigue that is focused on its severity. Advantageous of the VAS is that it is simple to administer. Also the testretest, construct validity and sensitivity to change in RA are reported to be good. ${ }^{17}$

Present study mainly focused on the longitudinal course of fatigue in patients with RA, though we also evaluated whether fatigue severity of patients with RA differed to fatigue experienced by patients with other forms of early arthritis. Patients with SCTD and RS3PE had significantly more severe fatigue and patients with UA, PsA/SpA or septic arthritis had significantly less fatigue compared to patients with RA. However, some of the observed differences were small and it can be questioned whether these differences are clinically relevant. The differences observed at baseline remained present over time. To the best of our best knowledge, this has not been reported on this scale previously.

To conclude, in this large longitudinal study on fatigue in RA, fatigue was a persistent problem, despite treatment. The median level of fatigue experienced by a population-based cohort of patients with RA remained even after many years of disease around $40 \mathrm{~mm}$ and the applied treatment strategies did not reduce fatigue levels. Therefore, as persistent fatigue is associated with functional loss, fatigue in RA remains an 'unmet need'.

Contributors HWvS and RT analysed the data. HWvS, TWJH, AB and AHMvdH-vM contributed to the conception, design and interpretation of the work. HWvS an AHMvdH-vM drafted the manuscript and all other authors revised the manuscript for important intellectual content. All authors gave final approval of the version to be published.

Funding This work was supported by a Vidi-grant of the Netherlands Organisation for Scientific Research, BTCURE and Masterswitch.

Competing interests None.

Patient consent Obtained.

Ethics approval Local medical ethical committee of Leiden University Medical Center.

Provenance and peer review Not commissioned; externally peer reviewed.

Data sharing statement No additional data are available.

Open Access This is an Open Access article distributed in accordance with the Creative Commons Attribution Non Commercial (CC BY-NC 4.0) license, which permits others to distribute, remix, adapt, build upon this work noncommercially, and license their derivative works on different terms, provided the original work is properly cited and the use is non-commercial. See: http:// creativecommons.org/licenses/by-nc/4.0/

\section{REFERENCES}

1. Hewlett S, Carr M, Ryan S, et al. Outcomes generated by patients with rheumatoid arthritis: how important are they? Musculoskeletal Care 2005;3:131-42.
2. Wolfe F, Hawley DJ, Wilson K. The prevalence and meaning of fatigue in rheumatic disease. J Rheumatol 1996;23:1407-17.

3. Pollard LC, Choy EH, Gonzalez J, et al. Fatigue in rheumatoid arthritis reflects pain, not disease activity. Rheumatology 2006;45:885-9.

4. Nikolaus S, Bode C, Taal E, et al. Fatigue and factors related to fatigue in rheumatoid arthritis: a systematic review. Arthritis Care Res 2013;65:1128-46

5. Breedveld FC, Han C, Bala M, et al. Association between baseline radiographic damage and improvement in physical function after treatment of patients with rheumatoid arthritis. Ann Rheum Dis 2005;64:52-5.

6. Wolfe F, Michaud K. Predicting depression in rheumatoid arthritis: the signal importance of pain extent and fatigue, and comorbidity. Arthritis Care Res 2009;61:667-73.

7. Lacaille D, White MA, Backman CL, et al. Problems faced at work due to inflammatory arthritis: new insights gained from understanding patients' perspective. Arthritis Care Res 2007;57:1269-79.

8. Kirwan JR, Minnock P, Adebajo A, et al. Patient perspective: fatigue as a recommended patient centered outcome measure in rheumatoid arthritis. J Rheumatol 2007;34:1174-7.

9. Aletaha D, Landewe R, Karonitsch T, et al. Reporting disease activity in clinical trials of patients with rheumatoid arthritis: EULAR/ ACR collaborative recommendations. Arthritis Care Res 2008;59:1371-7.

10. Hewlett $\mathrm{S}$, Chalder $\mathrm{T}$, Choy $\mathrm{E}$, et al. Fatigue in rheumatoid arthritis: time for a conceptual model. Rheumatology 2011;50: 1004-6.

11. van Hoogmoed D, Fransen J, Bleijenberg G, et al. Physical and psychosocial correlates of severe fatigue in rheumatoid arthritis. Rheumatology 2010;49:1294-302.

12. Davis MC, Zautra AJ, Younger J, et al. Chronic stress and regulation of cellular markers of inflammation in rheumatoid arthritis: implications for fatigue. Brain Behav Immun 2008;22:24-32.

13. Stebbings $\mathrm{S}$, Herbison $\mathrm{P}$, Doyle $\mathrm{TCH}$, et al. A comparison of fatigue correlates in rheumatoid arthritis and osteoarthritis: disparity in associations with disability, anxiety and sleep disturbance. Rheumatology 2010;49:361-7.

14. Thyberg I, Dahlström Ö, Thyberg M. Factors related to fatigue in women and men with early rheumatoid arthritis: the Swedish TIRA study. J Rehabil Med 2009;41:904-12.

15. Treharne GJ, Lyons AC, Hale ED, et al. Predictors of fatigue over 1 year among people with rheumatoid arthritis. Psychol Health Amp Med 2008:13:494-504.

16. de Rooy DPC, van der Linden MPM, Knevel R, et al. Predicting arthritis outcomes-what can be learned from the Leiden Early Arthritis Clinic? Rheumatology 2011;50:93-100.

17. Hewlett S, Dures E, Almeida C. Measures of fatigue: Bristol Rheumatoid Arthritis Fatigue Multi-Dimensional Questionnaire (BRAF MDQ), Bristol Rheumatoid Arthritis Fatigue Numerical Rating Scales (BRAF NRS) for Severity, Effect, and Coping, Chalder Fatigue Questionnaire (CFQ), Checklist Individual Strength (CIS20R and CIS8R), Fatigue Severity Scale (FSS), Functional Assessment Chronic Illness Therapy (Fatigue) (FACIT-F), Multi-Dimensional Assessment of Fatigue (MAF), Multi-Dimensional Fatigue Inventory (MFI), Pediatric Quality Of Life (PedsQL) Multi-Dimensional Fatigue Scale, Profile of Fatigue (ProF), Short Form 36 Vitality Subscale (SF-36 VT), and Visual Analog Scales (VAS). Arthritis Care Res 2011;63:S263-86.

18. Nicklin J, Cramp F, Kirwan J, et al. Measuring fatigue in rheumatoid arthritis: a cross-sectional study to evaluate the Bristol Rheumatoid Arthritis Fatigue Multi-Dimensional questionnaire, visual analog scales, and numerical rating scales. Arthritis Care Res 2010;62:1559-68.

19. Hjermstad MJ, Fayers PM, Haugen DF, et al. Studies comparing numerical rating scales, verbal rating scales, and visual analogue scales for assessment of pain intensity in adults: a systematic literature review. J Pain Symptom Manage 2011;41: 1073-93.

20. Geraci M, Bottai M. Linear quantile mixed models. Stat Comput 2014;24:461-79.

21. Geraci M. Linear quantile mixed models: the Iqmm package for Laplace quantile regression. J Stat Softw 2014;47.

22. Beyerlein A. Quantile regression-opportunities and challenges from a user's perspective. Am J Epidemiol 2014:kwu178.

23. van Buuren S, Groothuis-Oudshoorn K. Mice: multivariate imputation by chained equations in R. J Stat Softw 2011;45.

24. Knevel R, Tsonaka R, le Cessie S, et al. Comparison of methodologies for analysing the progression of joint destruction in rheumatoid arthritis. Scand J Rheumatol 2013;42:182-9. 
25. Knevel R, de Rooy DPC, Zhernakova A, et al. Association of variants in IL2RA with progression of joint destruction in rheumatoid arthritis. Arthritis Rheum 2013;65:1684-93.

26. Therneau TM, Grambsch PM. Modeling survival data: extending the cox model. Springer, 2000.

27. Therneau TM. A package for survival analysis in $S, R$ package version 2.37-7. 2014. http://CRAN.R-project.org/package=survival

28. Khanna D, Pope JE, Khanna PP, et al. The minimally important difference for the fatigue visual analog scale in patients with rheumatoid arthritis followed in an academic clinical practice. $J$ Rheumatol 2008;35:2339-43.

29. Putrik P, Ramiro S, Keszei AP, et al. Lower education and living in countries with lower wealth are associated with higher disease activity in rheumatoid arthritis: results from the multinational COMORA study. Ann Rheum Dis 2015. Published Online First: 17 Feb 2015. doi:10.1136/annrheumdis-2014-206737

30. Pincus T, Sokka T, Kautiainen H. Patients seen for standard rheumatoid arthritis care have significantly better articular, radiographic, laboratory, and functional status in 2000 than in 1985. Arthritis Rheum 2005:52:1009-19.

31. Cramp F, Hewlett S, Almeida C, et al. Non-pharmacological interventions for fatigue in rheumatoid arthritis. In: Cochrane Database of Systematic Reviews. John Wiley \& Sons, Ltd, 1996. http://onlinelibrary.wiley.com/doi/10.1002/14651858.CD008322.pub2/ abstract (accessed 14 July 2014).

32. Sokka T, Toloza S, Cutolo M, et al. Women, men, and rheumatoid arthritis: analyses of disease activity, disease characteristics, and treatments in the QUEST-RA Study. Arthritis Res Ther 2009; 11:R7.

33. Ahlmén M, Svensson B, Albertsson $\mathrm{K}$, et al. Influence of gender on assessments of disease activity and function in early rheumatoid arthritis in relation to radiographic joint damage. Ann Rheum Dis 2010;69:230-3.

34. Donders ART, van der Heijden GJMG, Stijnen T, et al. Review: a gentle introduction to imputation of missing values. J Clin Epidemiol 2006;59:1087-91. 